\title{
Terapia por láser de baja potencia: consideraciones óptico-físicas y biológicas determinantes en su aplicación clínica
}

\author{
M. Conde Quintero , J.C.G. de Sande y M.J. Toscano Arroyo
}

\begin{abstract}
Resumen
Objetivos: Analizar la distribución de energía en un tejido cuando se emplea terapia por láser de baja potencia y estudiar las especificaciones mínimas de equipos de terapia láser para estimar la dosis.

Material y métodos: Se ha empleado el método de Monte Carlo para obtener la distribución de energía absorbida por la piel para dos tipos de láser y la teoría de la difusión para estimar la longitud de penetración y el recorrido libre medio. Se ha estudiado la variación de esa distribución en función de la raza (caucásico, asiático, afroamericano) y para dos localizaciones anatómicas distintas. Se ha analizado la información facilitada por diversos fabricantes de equipos comerciales para determinar si es necesario adaptar la dosimetría recomendada. Resultados: La radiación láser infrarroja $(810 \mathrm{~nm})$ se absorbe mayoritariamente en un espesor de piel de $1,9 \pm 0,2 \mathrm{~mm}$ para caucásicos, entre $1,73 \pm 0,08 \mathrm{~mm}$ (volar del antebrazo) y $1,80 \pm 0,11 \mathrm{~mm}$ (palma) para asiáticos y entre $1,25 \pm 0,09 \mathrm{~mm}$ (volar del antebrazo) y $1,65 \pm 0,2 \mathrm{~mm}$ (palma) para afroamericanos. El recorrido libre medio de la luz siempre es menor que $0,69 \pm 0,09 \mathrm{~mm}$. Para los equipos comerciales analizados la única característica geométrica del haz láser que se menciona es la superficie que oscila entre 0,08 y $1 \mathrm{~cm}^{2}$, pero no se especifica cómo es la distribución de energía, la divergencia del haz, forma de la sección transversal, etc.

Conclusiones: Dependiendo del equipo de terapia por láser de baja potencia utilizado, el tipo de paciente y la zona a tratar, el clínico debe adaptar las dosis recomendadas.
\end{abstract}


Low power laser therapy: opto-physical and biological considerations underlying its clinical application

\begin{abstract}
Objectives: To analyze the distribution of energy deposited in a tissue when this is irradiated with a low power laser and to study the minimum characteristics that manufacturers of low power laser therapy equipments should include to estimate the dosage.

Material and methods: Monte Carlo simulation was performed to determine the absorption location of the laser energy. The diffusion theory was used to estimate penetration depth and mean free path. Variation of this distribution was studied based on three different skin types (Caucasians, Asians and Afroamericans) and for two different anatomic locations: palm and volar forearm. Information given by several manufactures of low power laser therapy equipments has been analyzed.

Results: Infrared $(810 \mathrm{~nm})$ laser radiation is mainly absorbed in a skin layer of thickness $1.9 \pm 0.2 \mathrm{~mm}$ for Caucasians, from $1.73 \pm 0.08 \mathrm{~mm}$ (volar forearm) to $1.80 \pm 0.11 \mathrm{~mm}$ (palm) for Asians, and from $1.25 \pm 0.09 \mathrm{~mm}$ (volar forearm) to $1.65 \pm 0.2 \mathrm{~mm}$ (palm) for Afroamericans. The light mean free path is lower than $0.69 \pm 0.09 \mathrm{~mm}$ for all cases. The laser beam characteristics (beam shape, energy distribution on a transversal section, divergence, incidence angle, etc.) are not usually specified by the manufacturers. Only beam size (ranging from 0.08 to $1 \mathrm{~cm}^{2}$ ) is given in some cases.

Discussion and conclusions: Depending on the low power laser therapy equipment, on the patient and on the anatomic area to be treated, the staff should adapt the recommended dosage for each individual case.
\end{abstract}

\section{Introducción}

Las aplicaciones terapéuticas del láser en las que el aumento local de temperatura es despreciable se engloban dentro de lo que se conoce como terapia por láser de baja potencia, de bajo nivel o bioestimulación por láser (LPLT o LLLT del inglés Low Power Laser Therapy o Low Level Laser Therapy, usaremos LPLT en lo sucesivo $)^{1,2}$. Aunque los efectos terapéuticos del láser de baja potencia están documentados ${ }^{3-7}$ aun no está claro cuál es el mecanismo, a nivel celular y orgánico, mediante el que se produce la acción terapéutica y cuáles son los parámetros óptimos de la luz que se debe emplear para cada aplicación clínica. Para que el láser produzca algún efecto sobre los tejidos es necesario que ocurra la absorción de la radiación láser y son las moléculas fotorreceptoras las que desencadenan todo un conjunto de respuestas celulares implicadas en estos fenómenos tisulares. Tras la absorción de la luz por algún cromóforo se pueden producir una serie de procesos que se dividen en radiativos y no radiativos ${ }^{1,2,8,9}$. En los procesos radiativos, los cromóforos, después de su excitación vuelven a su estado inicial debido a la emisión de luz. En los procesos no radiativos, la energía de los fotones absorbidos se convierte en otro tipo de energía, dando lugar a fenómenos fotoquímicos o térmicos ${ }^{8}$. En los fotoquímicos, se desencadenan diversas reacciones químicas entre las que podemos citar la fotoadición, fotofragmentación, fotooxidación, etc. En el caso de efectos térmicos, se produce un calentamiento de la zona irradiada y de las zonas adyacentes y puede dar lugar a una hipertermia, desnaturalización de proteínas, fotocoagulación, vaporización, carbonización, etc.

Los efectos de la LPLT son bioquímicos. Se trata de la fotobiomodulación ${ }^{1}$ o fotobioestimulación ${ }^{2,9}$ y consiste en el aumento del metabolismo celular bien por activación de la cadena respiratoria bien por activación de fotorreceptores no mitocondriales. Muchos de los componentes normales de la piel son cromóforos (hemoglobina, oxihemoglobina, bilirrubina, melanina, agua, etc.) y cada uno de ellos absorbe en mayor o menor medida cada longitud de onda ${ }^{2,9}$. Cuando la luz absorbida se transforma en energía química, modifica algún cromóforo de forma transitoria o permanente $y$ este hecho activa todos los procesos fotobiológicos inherentes a la LPLT. Se considera que el cromóforo que desencadena las distintas reacciones bioquímicas es el mismo y que estas respuestas se producen en varios orgánulos celulares (núcleo, plasma y membrana). Actualmente las investigaciones se centran en la enzima citocromo $c$ oxidasa, que forma parte de la etapa terminal de la cadena respiratoria celular ${ }^{1,2,10}$.

Los tejidos que fisiológicamente se encuentran en condiciones patológicas, con la consiguiente disminución del $\mathrm{pH}$ que ello supone, son más sensibles a la irradiación y por tanto su capacidad de activación y posterior respuesta a la aplicación del láser es mayor ${ }^{11}$.

En los primeros equipos de LPLT desarrollados se empleaba un láser de $\mathrm{He}-\mathrm{Ne}$ (visible) que emiten un haz con una distribución de energía en su sección transversal no uniforme (en el caso de láseres monomodo esa distribución es aproximadamente gaussiana). En la actualidad, debido al tamaño, bajo consumo, bajo coste, facilidad de fabricación, etc., se emplean láseres de diodo en los equipos de LPLT $^{12-19}$. Las longitudes de onda de emisión de los láseres de diodo barren casi todo el visible y el infrarrojo cercano ${ }^{2}$. Los láseres de diodo tienen dos inconvenientes: la distribución de la energía en su sección transversal puede ser 

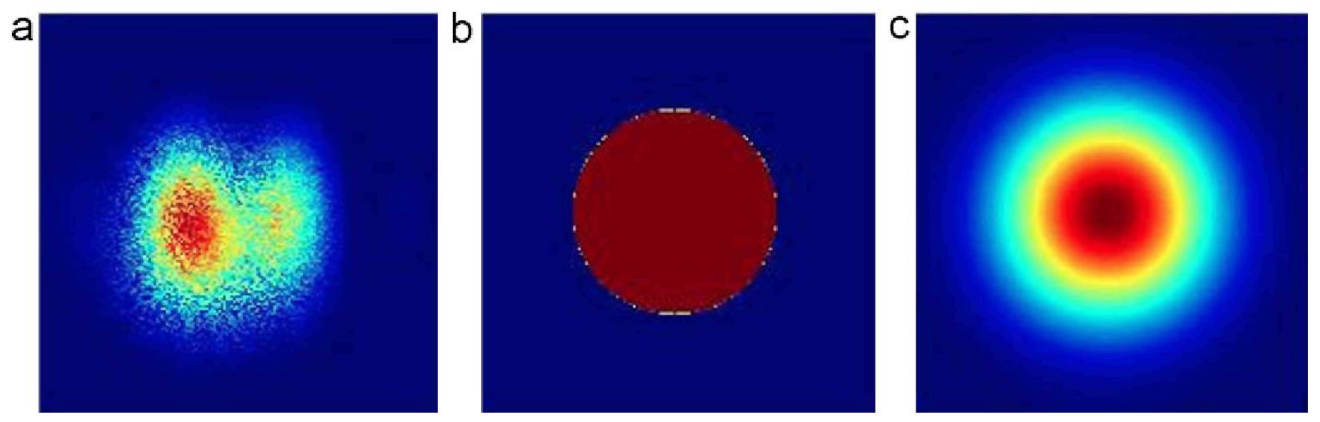

Figura 1 Ejemplos de distribución de energía en una sección transversal de un haz láser: a) haz de láser de diodo; b) haz uniforme; c) haz gaussiano.

muy irregular y se modifica cuando se propaga (fig. 1a) y la divergencia del haz es astigmática y mucho más grande que en otros tipos de láseres (este problema se puede corregir parcialmente mediante el uso de lentes asféricas). Los equipos de LPLT actualmente usados en fisioterapia establecen automáticamente el tiempo de aplicación a partir de la dosis requerida (energía total por unidad de área que debe alcanzar la superficie de la piel; en $\left.\mathrm{J} / \mathrm{cm}^{2}\right)^{12-19}$. Sin embargo, la cantidad de energía que se absorbe en la zona tratada varía, no solo con las características del emisor, sino también con el tipo de piel que estamos tratando.

En nuestra experiencia clínica nos encontramos ante el hecho de que, aplicando valores de dosimetría superiores a los que otros autores proponían para alcanzar un efecto terapéutico determinado, obteníamos un resultado mejor y de manera más rápida ${ }^{18-21}$.

Los objetivos de este trabajo son:

- Predecir la cantidad y localización de la energía absorbida para unas condiciones de aplicación de LPLT ideales para distintos tipos de piel.

- Definir la información mínima que debe suministrar el fabricante de equipos de LPLT para que el profesional clínico pueda conocer con la mayor precisión posible la dosis que realmente está suministrando.

\section{Material y método}

Por un lado, se han recopilado las características técnicas del láser empleado en diversos equipos comerciales: modo de funcionamiento, longitud de onda de emisión, potencia de emisión, sección del haz, divergencia del haz, distribución de la energía en la sección transversal, distancia desde el emisor a la salida del aplicador y posible óptica intermedia empleada, modo de aplicación del haz láser (en contacto con la piel o a una distancia que pueda ser variable), etc. La información se ha extraído de la descripción suministrada por los fabricantes/distribuidores de diversos equipos de $\mathrm{LPLT}^{16-18}$.

Por otro lado, se ha calculado cómo se distribuye la energía dentro de un tejido bajo unas condiciones ideales de irradiación. Cuando un haz de luz incide sobre un tejido biológico se producen una serie de fenómenos que modifican la distribución de energía en una sección transversal del haz. Los efectos biológicos que esa luz pueda producir dependen de dónde sea absorbida y de qué cromóforo la absorbe. La propagación de la luz a través de un material túrbido se puede describir mediante la ecuación de transferencia de la radiación?. Comúnmente se emplean dos métodos para resolver dicha ecuación: mediante simulación Monte Carlo o mediante la teoría de la difusión ${ }^{9,22}$.

\section{Método de Monte Carlo}

Para describir la propagación de la luz es necesario un modelo físico del tejido. Desde un punto de vista anatómico podemos describir la piel como una estructura de tres capas $^{23}$ : la epidermis ( $100 \mu \mathrm{m}$ de espesor), la dermis (entre 1 y $4 \mathrm{~mm}$ de espesor) y la capa adiposa (de 1 y $6 \mathrm{~mm}$ ). La absorción del conjunto es principalmente debida a la melanina (en la epidermis) a la oxihemoglobina, la deoxihemoglobina, carotenos y bilirrubina (en la dermis) y a la hemoglobina, los lípidos y el agua (en la capa adiposa). El esparcimiento viene determinado por las partículas de melanina por las fibras de colágeno y los adipocitos.

Cuando un haz de luz incide sobre la piel, una parte de la luz se refleja y otra se refracta y penetra en el tejido. En todo el rango visible el infrarrojo cercano la luz reflejada supone alrededor de un $5 \%$ de la luz incidente para ángulos de incidencia próximos a la normal ${ }^{9}$.

Dentro del tejido, la luz se absorbe, se esparce o se transmite. Los cromóforos de la piel absorben selectivamente parte de esa luz con un coeficiente de absorción $\mu_{a}$. El esparcimiento debido a inhomogeneidades del tejido queda descrito por un coeficiente de esparcimiento $\mu_{\mathrm{s}}$ y un parámetro de anisotropía, g. Parte de la luz esparcida puede alcanzar la superficie de la piel donde una parte se refractará hacia el exterior (reflexión difusa de la piel) y otra se reflejará hacia el interior. Debido a la absorción y esparcimiento, la energía por unidad de superficie (fluencia) que alcanza capas más profundas de la piel disminuye exponencialmente según la ley de Lambert-Beer. Para la piel en el rango visible e infrarrojo, la fluencia cae en 4 órdenes de magnitud en unos pocos $\mathrm{mm}^{24}$. Por último, una parte de la luz puede atravesar la piel y llegar a tejidos subcutáneos donde continúa produciéndose absorción y esparcimiento hasta que toda la luz sea finalmente absorbida o se transmita a través de todos los tejidos ${ }^{2,8,9}$.

Hemos considerado la piel como un medio homogéneo e isótropo caracterizado por su índice de refracción $(n=1,40$ en todos los $\operatorname{casos}^{23}$ ) su coeficiente de absorción $\left(\mu_{a}\right)$, su coeficiente de esparcimiento $\left(\mu_{s}\right)$ y el parámetro de anisotropía $(g)^{23}$. Hemos utilizamos el código de Monte Carlo desarrollado por Wang et $\mathrm{al}^{22}$ para obtener la energía absor- 
Tabla 1 Parámetros y resultados

\begin{tabular}{|c|c|c|c|c|c|c|}
\hline \multirow[t]{2}{*}{ Zona } & \multicolumn{2}{|c|}{ Caucásico } & \multicolumn{2}{|c|}{ Asiático } & \multicolumn{2}{|c|}{ Afroamericano } \\
\hline & Palma & Vda. & Palma & Vda. & Palma & Vda. \\
\hline$\mu_{a}\left(\mathrm{~cm}^{-1}\right)$ & $0,60 \pm 0,07$ & $0,57 \pm 0,1$ & $0,60 \pm 0,07$ & $0,60 \pm 0,04$ & $0,83 \pm 0,2$ & $1,5 \pm 0,5$ \\
\hline$\mu^{\prime}{ }_{s}\left(\mathrm{~cm}^{-1}\right)$ & $15,2 \pm 0,8$ & $16 \pm 1$ & $16,5 \pm 0,5$ & $18 \pm 1$ & $14 \pm 2$ & $13 \pm 2$ \\
\hline$R_{d}(\%)$ & 39,4 & 41,3 & 41,4 & 42,3 & 33,2 & 23,4 \\
\hline$A(\%)$ & 57,4 & 55,5 & 55,6 & 54,6 & 63,4 & 73,8 \\
\hline$T(\%)$ & 0,5 & 0,4 & 0,03 & 0,2 & 0,2 & 0,0 \\
\hline$\delta(\mathrm{mm})$ & $1,88 \pm 0,12$ & $1,88 \pm 0,2$ & $1,80 \pm 0,11$ & $1,73 \pm 0,08$ & $1,65 \pm 0,2$ & $1,25 \pm 0,08$ \\
\hline$r(m(\mathrm{~mm})$ & $0,64 \pm 0,03$ & $0,60 \pm 0,04$ & $0,57 \pm 0,02$ & $0,54 \pm 0,03$ & $0,67 \pm 0,09$ & $0,69 \pm 0,09$ \\
\hline
\end{tabular}

Coeficientes de absorción, $\mu_{a}$, y de esparcimiento reducido, $\mu_{\text {'s, empleados en la simulación }}^{9,26}$.

A: absorción total; $\delta$; longitud de penetración; $R_{d}$ : reflectancia difusa; $r$ Im: recorrido libre medio; vda: volar del antebrazo.

bida localmente en cada punto del medio cuando incide un haz de luz de anchura despreciable (toda la luz incide en un mismo punto) y con dirección de incidencia perpendicular a la superficie. En este código se tiene en cuenta la interacción en tres dimensiones de los fotones incidentes con el material. Como el problema tiene simetría de revolución respecto al eje del haz (siempre que incida en dirección normal a la superficie), la solución obtenida solo depende de la profundidad en el tejido y de la distancia desde el punto de incidencia. Posteriormente hemos realizado la convolución de esta solución con una distribución de energía uniforme o gaussiana (fig. 1b y 1c) para determinar cómo se absorbe la luz dentro del tejido cuando se incide con un haz de diámetro finito ${ }^{25}$. Finalmente se han representado mapas de la energía absorbida por unidad de volumen en función de la profundidad y de la separación desde el eje del haz láser.

Hemos tomado valores de los coeficientes de absorción y esparcimiento medidos para distintos tipos de piel ${ }^{24,25}$ a una longitud de onda de $810 \mathrm{~nm}$ y un haz láser con una sección circular de $1 \mathrm{~cm}^{2}$ de área (radio $0,56 \mathrm{~cm}$ ) y energía total $1 \mathrm{~J}$. Los valores de los parámetros se exponen en la tabla 1.

\section{Teoría de la difusión de la radiación}

La teoría de la difusión predice una disminución con la profundidad de tipo exponencial con una longitud de penetración $\delta$ (longitud que debemos profundizar en el tejido para que la energía disminuya en un factor 1/e), y un recorrido libre medio $\mathrm{rlm}$ (distancia promedio entre colisiones de un fotón con los centros dispersores o con los cromóforos) dados por ${ }^{2,22}$ :

$\delta=1 / \sqrt{3 \mu_{a}\left(\mu_{a}+\mu_{s}(1-g)\right)}$,

$r l m=1 /\left[\mu_{a}+\mu_{s}(1-g)\right]$.

Para la piel en el infrarrojo cercano, $\mu_{s}^{\prime}=\mu_{s}(\tilde{1}-g) \gg$ $\mu_{a}$, con lo cual la longitud de penetración viene determinada por el producto de los coeficientes de absorción y esparcimiento y toma valores del orden de $\mathrm{mm}$. El recorrido libre medio, dominado por el coeficiente de esparcimiento reducido, suele ser bastante menor (del orden de decenas o centenas de $\mu \mathrm{m}$ ). Un aumento del coeficiente de absorción implica un aumento de la energía absorbida en una capa más superficial, mientras que un aumento del coeficiente de esparcimiento implica que haya mayor cantidad de luz reflejada de manera difusa, y por tanto menor absorción. En dirección lateral, debido al esparcimiento también se propaga parte de la luz y alcanza regiones que no están siendo irradiadas ${ }^{23}$. Sin embargo, la energía disminuye debido a la acción conjunta de la absorción y el esparcimiento y es de esperar que la zona a la que llega luz no sea mucho mayor que un recorrido libre medio ${ }^{22,23}$. Esto supone un aumento de la superficie bajo la que se está absorbiendo luz. Este aumento es significativo para haces láser muy estrechos (diámetros del orden o menores que $1 \mathrm{~mm}$ ) pero es poco relevante en el caso de haces láser con diámetros del orden o superiores a $1 \mathrm{~cm}$.

\section{Resultados y discusión}

Los efectos terapéuticos de la LPLT dependen de la localización espacial y de la cantidad de energía absorbida por los tejidos a tratar. El coeficiente de absorción del tejido determina cuánta energía se absorbe y la longitud de penetración determina hasta qué profundidad penetra la luz. En dirección lateral, son el recorrido libre medio y la anisotropía del esparcimiento los que determinan cuál es el aumento de la superficie bajo la cual llega luz.

En la tabla 1 se resumen los valores de la reflectancia total, especular y difusa, de la absorción total (medidas ambas como proporción de energía que se refleja o absorbe respecto a la incidente), así como las longitudes de penetración y el recorrido libre medio (Ecs. 1 y 2) para un haz láser que incide perpendicularmente sobre un tejido de $5 \mathrm{~mm}$ de espesor, descrito por los parámetros ópticos incluidos en la tabla. La transmitancia a través de esa capa es despreciable en todos los casos.

La reflectancia difusa tiene un valor muy elevado y disminuye con el aumento del coeficiente de absorción. Por el contrario, la proporción de energía absorbida es mayor cuanto mayor sea el coeficiente de absorción. En todos los casos, la longitud de penetración es mayor que $1 \mathrm{~mm}$ con lo que la mayor parte de luz atraviesa la epidermis $(\sim 100 \mu \mathrm{m})$ y alcanza la dermis.

La figura 2 muestra mapas de energía absorbida por unidad de volumen en cada punto en función de la profundidad y de la distancia desde el eje del haz láser (haz circular y uniforme, véase figura 1b). Se aprecian pequeñas diferencias en la forma en que se distribuye la energía absorbida para la palma de la mano y la zona volar del antebrazo de caucásicos. Esto mismo ocurre cuando se realiza el cálculo 

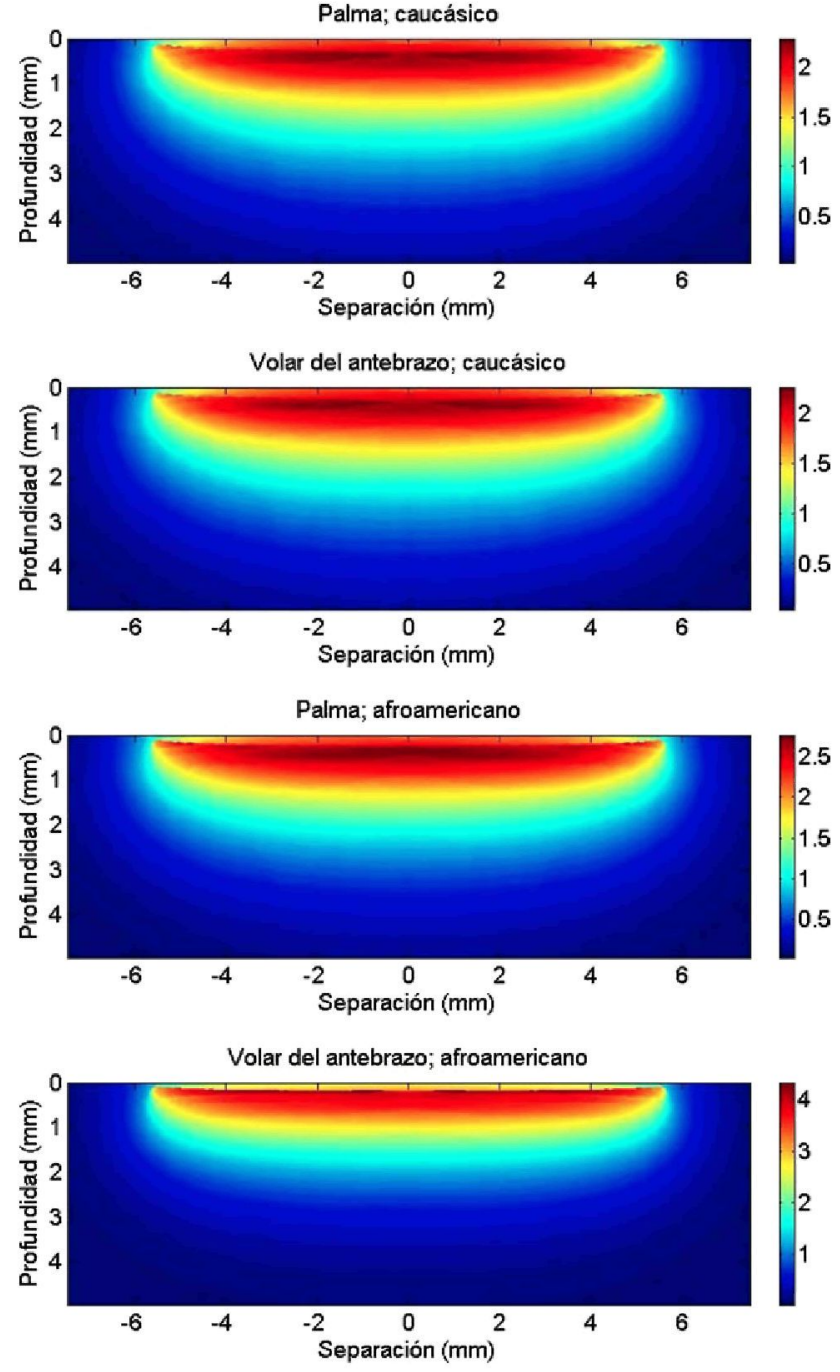

Figura 2 Energía absorbida por unidad de volumen (escala de color a la derecha, en $\mathrm{J} / \mathrm{cm}^{3}$ ) en función de la profundidad y de la separación desde el eje de un haz uniforme de radio $5,6 \mathrm{~mm}\left(1 \mathrm{~cm}^{2}\right.$ de área) y $1 \mathrm{~J}$ de energía que incide perpendicularmente en un tejido con los parámetros ópticos promedio correspondientes a la palma y al volar del antebrazo de 5 individuos caucásicos o para 5 individuos afroamericanos en cada caso.

para asiáticos con muy pocas diferencias numéricas (estos resultados no se muestran en la figura). Cuando se realiza el cálculo para afroamericanos se observa que hay una mayor absorción en el caso de la zona volar del antebrazo (valores más altos para la escala de color) y que esa absorción se concentra más cerca de la superficie (menor longitud de penetración). En los datos correspondientes de la tabla 1 se observa que la energía absorbida pasa de un 63,4 a un 73,8\%. Esto es lógico puesto que la zona volar del antebrazo, en el caso de afroamericanos, es más oscura (mayor pigmentación y mayor concentración de melanina). Comparando los resultados obtenidos en la palma pero para distinta raza, los resultados para caucásicos y asiáticos son casi idénticos y bastante parecidos con los obtenidos para afroamericanos (en este último caso se aprecia que la absorción es más superficial, compárese la primera y tercera imagen de la figura 2). En el caso de la zona volar del antebrazo se aprecia una diferencia notable entre los resultados obtenidos para caucásicos y para afroamericanos (compárese la segunda y cuarta imagen de la figura 2). Este resultado es lógico, pues se obtiene una longitud de penetración muy similar para todos los casos $(1,65 \mathrm{~mm}<\delta<1,89 \mathrm{~mm})$ menos el correspondiente al caso del volar del antebrazo para afroamericanos para los que esa longitud de penetración es sensiblemente menor $(\delta=1,25 \mathrm{~mm})$. En todos los casos se observa que el esparcimiento lateral es muy pequeño: desde los 5,6 mm de radio donde todavía se alcanzan valores muy altos de energía absorbida (color rojo) se pasa a valores muy pequeños (color azul) antes de llegar a los $6 \mathrm{~mm}$, lo cual es de esperar dado que el recorrido libre medio para todos los casos está entre 0,5 y $0,7 \mathrm{~mm}$ (tabla 1). Esto significa que el esparcimiento no da lugar a un aumento significativo de la superficie en la que se absorbe la luz siempre que el haz tenga un radio muy superior al recorrido libre medio, como es el caso de estas simulaciones (radio 5,6 mm).

En la figura 3 se representan simulaciones análogas a las de las figura 2 pero cambiando el tipo de haz. Ahora se incide con un haz de tipo gaussiano (ver figura 1c) con una energía total de $1 \mathrm{~J}$ y $5,6 \mathrm{~mm}$ radio (entendiendo por radio la distancia desde el eje para la cual la energía disminuye en $1 / \mathrm{e}^{2}$ ). La zona espacial en la que se absorbe la energía es muy similar en todos los casos salvo para el caso de la zona volar del antebrazo de afroamericanos.

Resulta llamativo que la distribución de la energía absorbida en la figura 3 es muy diferente a esa distribución en la figura 2. Hay una concentración mayor de energía absorbida en la zona central del haz donde se alcanzan energías mayores que para el caso de haz uniforme: el valor máximo de la energía supera los $6,5 \mathrm{~J} / \mathrm{cm}^{3}$ para el volar del antebrazo de afroamericanos en la parte central del haz gaussiano (cuarta imagen de la figura 3; haz gaussiano) mientras es poco más de $4 \mathrm{~J} / \mathrm{cm}^{3}$ para la misma raza y zona anatómica para haz uniforme (cuarta imagen de la figura 2, haz uniforme). Algo similar ocurre para las otras razas y zonas anatómicas. Es decir, que la forma en que se distribuye la energía en el haz incidente tiene una influencia muy grande en cómo distribuye la energía absorbida dentro del tejido a tratar. Esta influencia es mucho más importante que los cambios que se producen cuando se irradian distintas zonas de un mismo individuo o individuos de distintas razas (salvo casos extremos: palma de la mano y antebrazo en el caso de afroamericanos, o distintas razas). Debemos notar que la dispersión de valores medidos de los coeficientes de absorción y esparcimiento en una misma raza es muy grande $\mathrm{e}^{23-27}$. Por ejemplo, Chan et $\mathrm{al}^{27}$ obtienen valores de $\mu_{a}$ entre 0,2 y $1,3 \mathrm{~cm}^{-1}$ y valores de $\mu_{s}$ entre $6,9 \mathrm{y}$ $19,6 \mathrm{~cm}^{-1}$ para 6 individuos caucásicos. Eso significaría que entre esos individuos podría haber diferencias tan grandes en la absorción total como la mostrada en la tabla 1 y diferencias tan grandes en la distribución de la energía absorbida como las mostradas entre la primera y la cuarta imagen de la figura 2.

Debido a la variación con la longitud de onda de los parámetros ópticos, estos resultados son extrapolables a otras longitudes de onda de manera cualitativa; es decir, la longitud de penetración y/o el recorrido libre medio pueden variar considerablemente con la longitud de onda, pero una vez fijada la longitud de onda, la forma en que se distribuye 

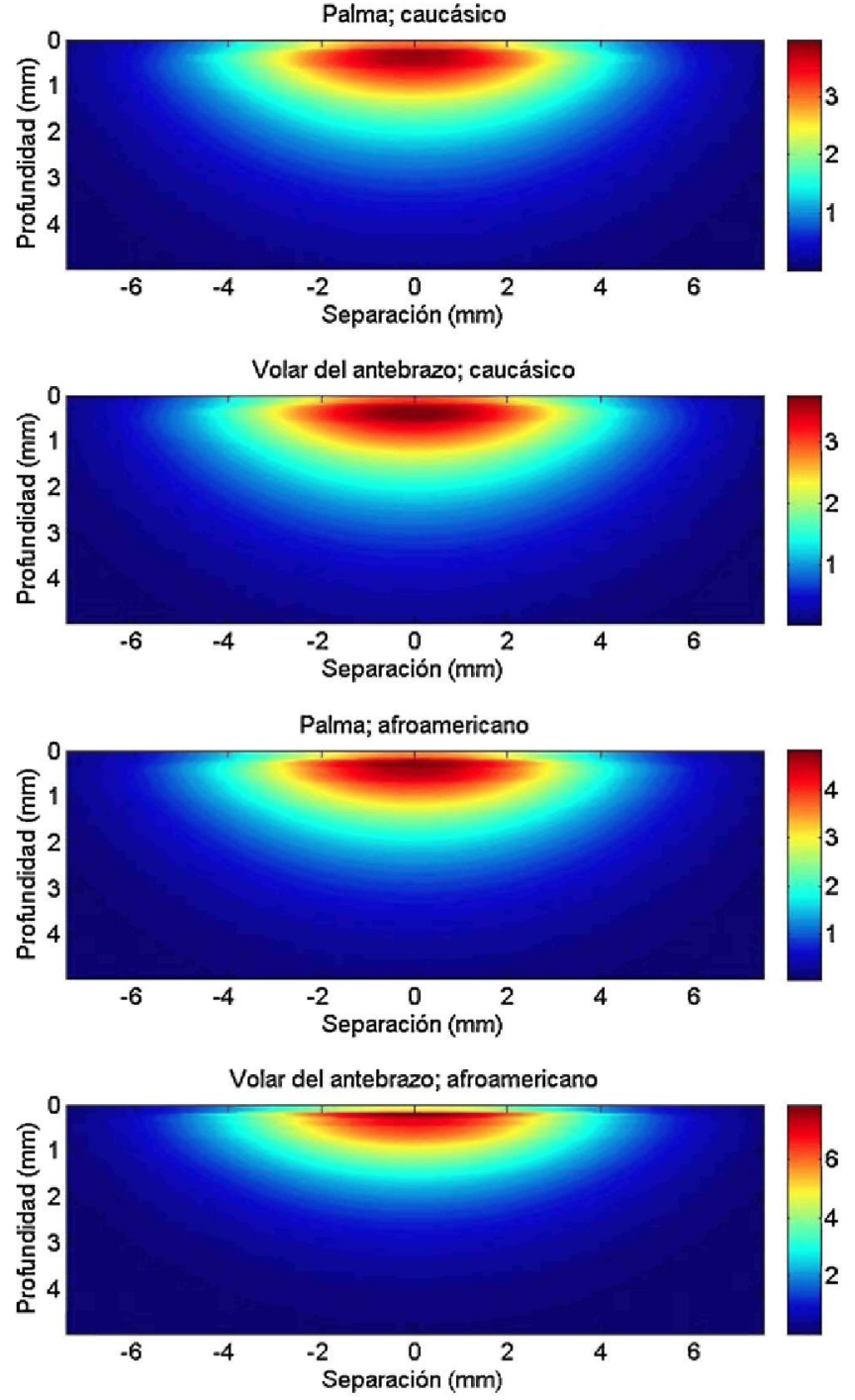

Figura 3 Energía absorbida por unidad de volumen (escala de color a la derecha, en $\mathrm{J} / \mathrm{cm}^{3}$ ) en función de la profundidad y de la separación desde el eje de un haz gaussiano de radio $5,6 \mathrm{~mm}\left(1 \mathrm{~cm}^{2}\right.$ de área) y $1 \mathrm{~J}$ de energía que incide perpendicularmente en un tejido con los parámetros ópticos promedio correspondientes a la palma y al volar del antebrazo de 5 individuos caucásicos o para 5 individuos afroamericanos en cada caso.

la absorción de energía en el tejido varía tanto al cambiar el tipo de haz como al cambiar el tipo de piel.

Teniendo en cuenta las simulaciones (figs. 2 y 3) queda claro que para facilitar el trabajo del fisioterapeuta que aplica LPLT, sería conveniente el uso de láseres cuyo haz tuviera una distribución de energía lo más uniforme posible en su sección transversal. En ese caso y repitiendo aplicaciones sucesivas hasta cubrir toda la superficie de tejido a tratar, el clínico puede estar seguro de que está realizando una aplicación aproximadamente uniforme de la energía láser.

En el caso de haces gaussianos, el fisioterapeuta debe estimar (a partir de los datos de radio, diámetro o superficie del haz) cuanto debe solapar las aplicaciones sucesivas para obtener una distribución aproximadamente uniforme ${ }^{20}$.
Como en este caso se produce una mayor fluencia en la parte central del haz, también debe tener precaución para no dar lugar a una dosis excesiva o incluso a un calentamiento excesivo de la zona irradiada que pudiera ser lesivo.

Para el caso de haces láser con una distribución irregular de la energía en su sección transversal, como suelen ser los láseres de diodo, el fisioterapeuta se encuentra con una herramienta con la que tendrá que experimentar y aprender de su propia experiencia con el equipo empleado.

Teniendo en cuenta la absorción de luz por tejidos con distintos parámetros ópticos (tabla 1), si se pretende un tratamiento muy superficial, el fisioterapeuta tiene que estimar un aumento (para piel más clara) o una disminución (para piel más pigmentada) de la dosis recomendada debido a la menor o mayor absorción que presentan distintos tipos de piel. Esta regla se invierte si el tratamiento de LPLT pretende afectar a capas más profundas de tejido, puesto que la absorción es más superficial en tejidos más pigmentados. En este último caso no se deben sobrepasar valores de irradiación que puedan dañar las capas más superficiales de la piel.

La tabla 2 resume las características técnicas de los láseres de equipos de LPLT que se han recopilado a partir de la información disponible en las paginas web de diversos fabricantes y/o distribuidores ${ }^{16-18}$. Todos indican la longitud de onda de trabajo (nos hemos centrado en aquellos equipos que emplean longitudes de onda en el infrarrojo cercano, preferiblemente en $810 \mathrm{~nm}$ ), la potencia (en modo continuo) o potencia media (en modo pulsado) y el tipo de aplicación (pistola, lápiz a una determinada distancia). Solamente en algunos productos se encuentra información del área del haz láser (o de la energía o potencia por unidad de área suministrada) y este dato es fundamental para la aplicación de la LPLT. En ningún caso se especifica ni cómo es la distribución de la energía o potencia en una sección transversal del haz láser. Tampoco indican que se utilice algún elemento óptico entre el emisor y la salida del aplicador. En todos estos equipos se emplea un láser de diodo que normalmente tienen una distribución irregular de energía en la sección transversal del haz, y además, suelen presentar una sección no circular.

En algún caso el fabricante indica que la aplicación debe ser por contacto (tabla 2). Este modo de aplicación no es posible para el tratamiento de heridas abiertas, en cuyo caso se debe mantener el aplicador a una cierta distancia para evitar el contacto y consiguiente dolor del paciente $e^{20}$. Si se estuviera trabajando con láseres distintos a los de diodo, esta separación no supondría ningún problema dado que se puede asumir que la divergencia del haz es muy pequeña y por tanto la superficie sobre la que se reparte el haz no varía en unos pocos $\mathrm{cm}$ de propagación. Pero en el caso de los láseres de diodo la divergencia del haz puede ser muy grande $\left(8-10^{\circ}\right.$ en una dirección y $30-35^{\circ}$ en otra) si no se corrige mediante lentes asféricas. Aun en ese caso, algunos fabricantes que utilizan láseres con potencias relativamente altas, incluyen en el aplicador una lente divergente para evitar posibles daños debido a la exposición accidental del paciente o el clínico. En este caso también es necesario conocer la divergencia del haz láser para poder estimar cuál es el área que se está irradiando y con ello saber la energía por unidad de superficie que se le ha suministrado al paciente, cuando se separa el aplicador de la superficie. 
Tabla 2 Características de equipos LPLT

\begin{tabular}{|c|c|c|c|c|c|c|c|}
\hline Equipo & Tipo & l.d.o. $\mathrm{nm}$ & $P \mathrm{~mW}$ & $f \mathrm{~Hz}$ & $E / t \mathrm{~J} / \mathrm{min}$ & $A \mathrm{~cm}^{2}$ & Aplicación \\
\hline \multirow[t]{2}{*}{ Megasonic 680 (a) } & Consola & 810 & 800 & $0,2-5 k$ & 48 & 1 & Pistola \\
\hline & & 658 & 40 & $0,8-5 k$ & 2,4 & 0,08 & Lápiz \\
\hline \multirow[t]{2}{*}{ Megascan 690 (a) } & Cañón & 810 & 1.500 & - & 90 & 0,5 & A $60 \mathrm{~cm}$ \\
\hline & & 658 & 25 & - & 1,5 & 0,12 & A $60 \mathrm{~cm}$ \\
\hline \multirow[t]{2}{*}{ BTL (b) } & Consola & 685 & $30-50$ & $0-10 \mathrm{k}$ & $1,8-3$ & Ajustable & Lápiz \\
\hline & & 830 & $50-400$ & $0-10 k$ & $3-24$ & Ajustable & Lápiz \\
\hline \multirow[t]{2}{*}{ Easyone (b) } & Cañón & 810 & $1.000-3.000$ & - & $60-180$ & - & A distancia \\
\hline & & 975 & 5.000 & - & 300 & - & A distancia \\
\hline \multirow[t]{2}{*}{ Idea terza (c) } & Consola & 808 & $500-2.000$ & $0,01-2 k$ & $30-120$ & - & Lápiz \\
\hline & & 905 & $12 \circ 24$ & $0,01-10 \mathrm{k}$ & $0,7-1,44$ & - & Lápiz \\
\hline Comby (c) & Cañón & 808 & $500-6.000$ & $0,01-2 k$ & $30-360$ & - & A distancia \\
\hline
\end{tabular}

$A$ : área; $E / t$ energía en un minuto; $f$ : frecuencia en régimen pulsado; l.d.o.: longitud de onda; $P$ : potencia (en continuo) o potencia media (en régimen pulsado).

Equipos de (a) Elecromedicarin, (b) Sanro y (c) Enraf-nonius ${ }^{12}$.

El fisioterapeuta, que se enfrenta a cifras dosimétricas basadas en datos empíricos obtenidos en laboratorio sobre muestras de tejidos o suspensiones celulares in vitro, debe tener presente estos condicionantes a la hora de traducirlas a la aplicación clínico ${ }^{28}$. Para una misma longitud de onda, la variación de los coeficientes de absorción y esparcimiento podría llevar a la variación de un $50 \%$ en la energía absorbida, lo que concuerda con la tabla de dosimetría antiinflamatoria propuesta por la World Associaton for Laser Therapy ${ }^{21}$. La dosis recomendada varía en un $\pm 50 \%$ de la fluencia $\left(\mathrm{J} / \mathrm{cm}^{2}\right)$ para obtener el mismo efecto terapéutico, en distintas localizaciones anatómicas para un mismo paciente. Además, para la misma localización pero distintos pacientes, también se establece una horquilla dosimétrica del $\pm 50 \% 21$. Incluso otros autores, tras afirmar que internacionalmente se establece como promedio el rango de 1 a $3 \mathrm{~J} / \mathrm{cm}^{2}$ para obtener un efecto antiinflamatorio con el láser, sostienen posteriormente que deben aplicar entre 3 y $5 \mathrm{~J} / \mathrm{cm}^{2}$ con su generador para obtenerlo ${ }^{12-20}$.

Conviene remarcar que los fototipos más comúnmente encontrados en nuestro ámbito terapéutico poblacional son el III y el IV ${ }^{29}$, factor que influiría también decisivamente en la fluencia necesaria para obtener un aporte energético terapéuticamente eficaz. Histológicamente se demuestra que la piel más pigmentada dispersa la acción del láser en la capa basal de la epidermis, mientras que la menos pigmentada presenta unos efectos a mayor profundidad y más circunscritos a la zona de aplicación de la luz $z^{30}$.

\section{Conclusiones}

En la aplicación clínica de LPLT, para conseguir una dosificación correcta es necesario tener en cuenta:

- El equipo concreto con el que se trabaja, que determinará la distribución de energía en una sección transversal del haz láser empleado, así como su potencia media o su energía por pulso, su área, divergencia etc. Sería recomendable que el fabricante/distribuidor del equipo suministrara información detallada sobre estas características, sobre todo área y divergencia, o al menos el área a las distancias del aplicador a las que se recomienda su utilización.

- El área bajo la cual se absorbe la luz coincide con el área del haz siempre que esta sea grande $\left(1 \mathrm{~cm}^{2}\right.$ o mayor).

- El área alcanzada por la radiación es mayor que el área del haz cuando ésta sea pequeña $\left(0,1 \mathrm{~cm}^{2}\right.$ o menor).

- Las características de la piel a tratar: su mayor o menor pigmentación, localización, etc.

\section{Conflicto de intereses}

Los autores declaran no tener ningún conflicto de intereses.

\section{Bibliografía}

1. Karu Tl. Low-power laser therapy. En: Vo-Dinh T, editor. Biomedical Photonics Handbook. Boca Raton: CRC Press; 2002. p. 1-25.

2. Waynant RW. Lasers in Medicine. Boca Raton: CRC Press; 2002.

3. Samson DJ, Lefevre F, Aronson N. Wound-healing technologies: low-level laser and vacuum-assisted closure. Evidence Report/Technology Assessment No. 111. (Prepared by the Blue Cross and Blue Shield Association Technology Evaluation Center Evidence-based Practice Center, under Contract No. 290-020026.) AHRQ Publication No. 05-E005-2. Rockville, MD: Agency for Healthcare Research and Quality. 2004.

4. Enwemeka CS, Parker JC, Dowdy DS, Harkness EE, Sanford LE, Woodruff LD. The efficacy of low-power lasers in tissue repair and pain control: a meta-analysis study. Photomed Laser Surg. 2004;22:323-9.

5. Simon, A. Low level laser therapy for wound healing: an update. Alberta Heritage Foundation for Medical Research; 2004 Oct. IP22 Information paper.

6. Orellana A, Hernández A, Larrea PJ, Fernández S, González $B M$. Láser infrarrojo frente a acupuntura en el tratamiento del espolón calcáneo. Rev Soc Esp Dolor. 2010;17:69-77.

7. Fernández R, Patiño S, Martínez A, Viñas $S$, Paseiro $G$, Barcia M. Analgesia por medios físicos en la patología de la ATM. Fisioterapia. 2003;25:293-305.

8. Prasad PN. Introduction to biophotonics. Hoboken: John Wiley \& Sons; 2003. 
9. Splinter R, Hooper BA. An introduction to biomedical optics. Boca Raton: Taylor \& Francis Group; 2007.

10. Karu TI, Pyatibrat LV, Afanasyeva NI. Cellular effects of low power laser therapy can be mediated by nitric oxide. Laser Surg Med. 2005;36:307-14.

11. Karu TI. The Science of Low Power Laser Therapy. London: Gordon and Breach Sci. Publ; 1998.

12. Laser Therapeutics, Inc. US Site [sede Web]. [acceso 12 de julio de 2010]. Disponible en: http://www. laserhealthsystems.com.

13. Cold laser [sede Web] Dallas, TX [acceso 12 de julio de 2010]. Disponible en: http://www.coldlaser.com.

14. Erchonia Laser Healthcare [sede web] [acceso 12 de julio de 2010]. Disponible en: http://www.erchonia.com.

15. Thor The Future of Medicine [sede Web] [acceso 12 de julio de 2010]. Disponible en: http://www.thorlaser.com.

16. Sanro electomedicina, [sede Web] [acceso 12 de julio de 2010]. Disponible en: http://www.sanro.com/.

17. Enraf Nonius [sede Web] [acceso 12 de julio de 2010]. Disponible en http://www.enraf.es/.

18. Carin-electromedicarin, S.A. [sede Web] [acceso 12 de julio de 2010]. Disponible en: http://www.electromedicarin.com.

19. TECE, S.A. Tecnología especializada [sede Web] [acceso 12 de julio de 2010]. Disponible en: http://www.tece.cu/.

20. Conde M, Toscano MJ, Estévez E. Tratamiento de heridas con láser infrarrojo. Aproximación a la dosis ideal. Cuestiones de fisioterapia. 2009;38:25-33.

21. World association for laser therapy [sede web]. [acceso 12 de julio de 2010]. Disponible en: http: //www. walt.nu/dosagerecommendations.html.
22. Wang $L$, Jacques SL, Zheng L. MCML-Monte Carlo modelling of photon transport in multi-layered tissues. Comput Methods Programs Biomed. 1995;47:131-46.

23. Bashkatov AN, Genina EA, Kochbey, Tuchin WV. Optical properties of human skin, subcutaneous and mucous tissues in the wavelength range from 400 to $2000 \mathrm{~nm}$. J Phys D: Appl Phys. 2005;38:2543-55.

24. Tseng SH, Bargo P, Durkin A, Kollias N. Chromophore concentrations, absorption and scattering properties of human skin in-vivo. Opt Express. 2009;17:14599-617.

25. Wang $L$, Jacques $S L$, Zheng $L$. CONV-convolution for responses to a finite diameter photon beam incident on multi-layered tissues. Compud Methods Programs Biomed. 1997;54:141-50.

26. Tseng SH, Grant A, Durkin A. In vivo determination of skin nearinfrared optical properties using diffuse optical spectroscopy. J Biomed Opt 2008;13:014016. doi:10.1117/1.2829772.

27. Chan EK, Sorg B, Protsenko D, O'Neil M, Motamedi M, Welch AJ. Effects of compression on soft tissue optical properties. IEEE J Sel Topics Quantum Electron. 1996;2:943-50.

28. Pozza DH, Fregapani PW, Weber JBB, Oliveira MG, Oliveira MAM, Ribeiro-Neto N, et al. Analgesic action of laser therapy (LLLT) in an animal model. Med Oral Patol Oral Cir Bucal. 2008;13:E648-52.

29. Consellería de Sanitat de la Generalitat Valenciana: Manual de prevención y tratamiento del melanoma. Valencia. 1999. I.S.B.N.84-482-1980-5.

30. Tan OT, Kerschmann R, Parrish JA. The effect of epidermal pigmentation on selective vascular effects of pulsed laser. Laser Surg Med. 1984;4:365-74. 